\title{
Did breast surgery change according to the guidelines? An internal audit focusing on the surgical outcome
}

\author{
Ebner $\mathrm{F}^{1 ; 2}$; Vasilic $\mathrm{D}^{1}$; Türck L $\mathrm{L}^{1}$; Baier $\mathrm{B}^{1}$; Albrecht $\mathrm{S}^{2}$
}

\author{
${ }^{1}$ HELIOS-Amper Klinikum, Dachau, Germany \\ 2Universität Ulm, Dachau, Germany
}

\section{Problem statement}

Over the last decade the recommendation for the tumor free margins has come down from $10 \mathrm{~mm}$ to 'no ink on tumor'. A similar development can be seen with the axillary lymphnode dissection (ALNE). At the end of the last millenium more removed lymph nodes were thought to be more beneficial to the survival of the patient. The introduction of the sentinel node biopsy (SNB) and further studies resulted in the current discussion if an ALNE is still necessary in certain circumstances.

Our internal audit analysed over time the surgical quality variables 'tumor free margins', 're-resection rate' and 'number of lymphnodes for SNB and ALNE'.

\section{Methods}

Retrospektive database analysis over a 10year period in our breast centre. Inclusion criteria were primary diagnosed breast cancer, final R0 resection and breast conserving surgery.

\section{Results}

Figure 1 provides information regarding the number of cases, R1-rate and average tumor free margin over time. The tumor free margin increases over the first years as the gold standard was $10 \mathrm{~mm}$ but consecutively drops as publications and guidelines reduce.

The rate of $\mathrm{R} 1$ resection peaks parallel to the tumor free margins and then also comes down to around $20 \%$ which is about the average over the time period.

The rate of SNB increased also parallel with the broadening indication up to $63 \%$ (Figure 2). The conversation rate of SNB to ALNE drops in the last 2 years.

The number of removed lymphnodes - as marker for long term post surgical problems - has been stable for SNB (min 1,8 LK; max 2,4 LK) and reduced over time for ALNE (min 11 (2013); $\max 20,6 \mathrm{LK}(2006))$ as shown in figure 2.

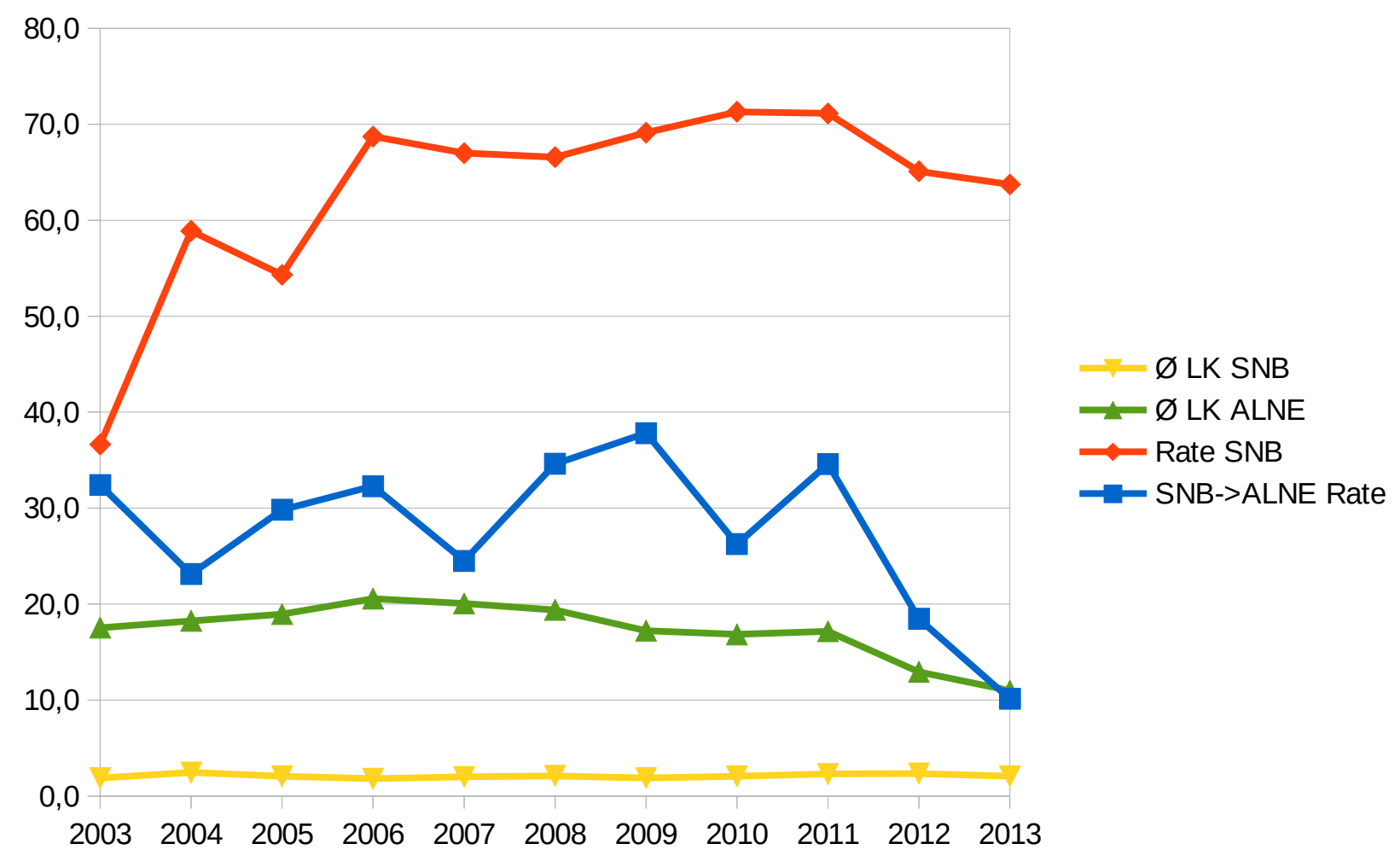

Figure 2: Overview of lymphnode variables with graphics for rate of SNB (\%), number of removed LN at SNB (Ø LK SNB), at axillary dissection (Ø LK ALNE) and rate of positive $L N$

leading to an ALNE (\%; SNB->ALNE).

\section{Diskussion}

The implementation of surgical guidelines can be seen over time in the annual statistic of our breast center. Parallel to the recommendations the tumor free margin doubled and then dropped again. The increase of the tumor free margin triggered a higher re-resection rate to fullfill this criteria. Currently with 'noink-on-tumor' the re-resection rate is on the long term average. More importaintly from a patients viewpoint in terms of complications is the stable number of removed SN over time. This shows that $\mathrm{SN}$ can be identified clearly and regularly. Parallel to the current scientific discussion our data shows that surgeons have started to become less radical in ALNE. Interestingly the rate of SNB conversion to ALNE also drops which may be due to the improved diagnosis in axillary ultrasound and core needle biopsy.

\section{Conclusion}

Our data shows that current surgical guidelines are slowly implemented in clincial day care and the effects can be seen over time. The next step should be the evaluation of the time from publication of new guidelines to the clinical implementation.

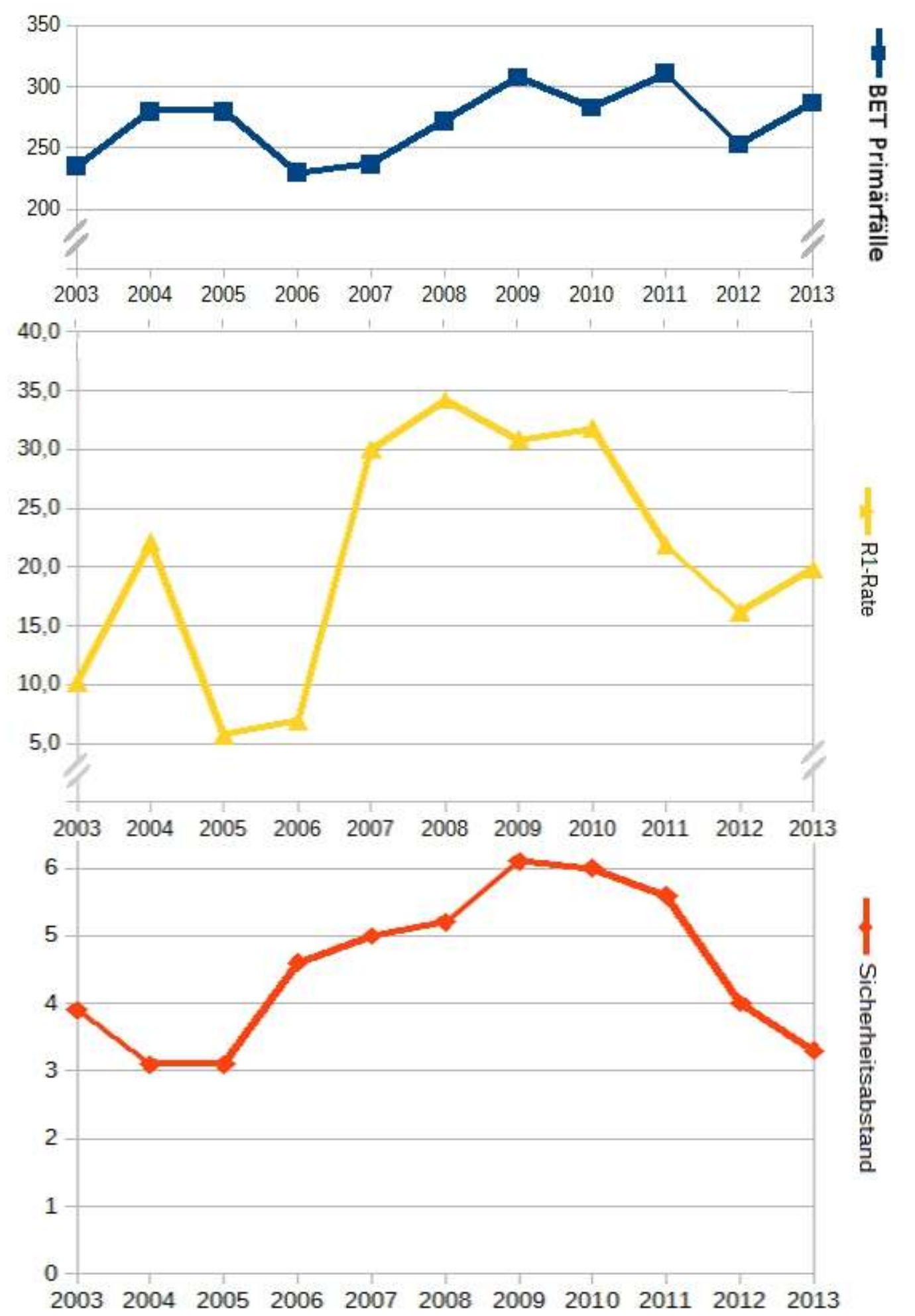

Figure 1: Overview of the variables over time with number of primary breast conserving breast cancers (BET-Fälle), R1-rate after primary surgery (\%) and average tumor free margin ( $\mathrm{mm})$. 